\author{
Charles D. Gomersall \\ Gavin M. Joynt \\ Philip Lam \\ Thomas Li \\ Florence Yap \\ Doris Lam \\ Thomas A. Buckley \\ Joseph J. Y. Sung \\ David S. Hui \\ Gregory E. Antonio \\ Anil T. Ahuja \\ Patricia Leung
}

Received: 4 August 2003

Accepted: 10 December 2003

Published online: 23 January 2004

(C) Springer-Verlag 2004

Electronic Supplementary Material Supplementary material is available in the online version of this article at http://dx.doi.org/10.1007/10.1007/00134003-2143-y

C. D. Gomersall ( • G. M. Joynt

P. Lam · T. Li · F. Yap - D. Lam ·

T. A. Buckley $\cdot$ P. Leung

Department of Anaesthesia

and Intensive Care,

The Chinese University of Hong Kong,

Prince of Wales Hospital,

Shatin, Hong Kong

e-mail: gomersall@cuhk.edu.hk

Tel.: +852-26322735

Fax: +852-26372422

J. J. Y. Sung · D. S. Hui

Department of Medicine and Therapeutics,

The Chinese University of Hong Kong,

Prince of Wales Hospital,

Shatin, Hong Kong

G. E. Antonio - A. T. Ahuja

Department of Diagnostic Radiology

and Organ Imaging,

The Chinese University of Hong Kong,

Prince of Wales Hospital,

Shatin, Hong Kong

\section{Short-term outcome of critically ill patients with severe acute respiratory syndrome}

\begin{abstract}
Objective: To document the outcome and determine prognostic factors for patients with severe acute respiratory syndrome who require admission to an intensive care unit. Design: Observational cohort study involving retrospective analysis of demographic, clinical, laboratory and radiological data. Setting: Adult intensive care unit in a tertiary referral university hospital involved in
\end{abstract} a major outbreak of severe acute respiratory syndrome (SARS).

Patients: The first 54 patients admitted with SARS to an intensive care unit (ICU). All were treated with corticosteroids, ribavirin, broad spectrum antimicrobials and supportive therapy. Interventions: None. Measurements and results: All patients were admitted for respiratory failure. The median APACHE II score was 11 (interquartile range 813). At 28 days 34 patients $(63 \%$; 95\% CI 49.6-74.6) were alive and not mechanically ventilated. Six patients were alive but ventilated (11.3\%; 95\% confidence interval 5.3-22.6) and 14 had died (25.9\%; CI 16.1-38.9). Seven of 27 ventilated patients developed evidence of barotrauma (25.9\%; 95\% CI 13.2-44.7). Median maximal multiple-organ dysfunction score was 5 (interquartile range 3.3-9). Median maximal respiratory dysfunction score was 3 (interquartile range 3-4). Increased age, severity of illness, lymphocyte count, decreased steroid dose, positive fluid balance, chronic disease or immunosuppression and nosocomial sepsis were associated with poor outcome on univariate analysis. Poor outcome was defined as death or need for mechanical ventilation at 28 days after ICU admission. Conclusions: Mortality amongst critically ill patients with SARS is high. It causes predominantly severe respiratory failure, with little other organ failure, and a high incidence of barotrauma amongst those requiring mechanical ventilation.

Keywords Coronavirus infections . Barotrauma - Critical care . Pneumonia, viral · Morbidity · Mortality

\section{Introduction}

Severe acute respiratory syndrome (SARS) is a new infectious disease that has rapidly spread around the world [1]. The causative agent is the new SARS coronavirus $[2,3,4]$. The virus is readily transmissible and the resulting disease clinically severe, with approximately $20 \%$ of cases requiring intensive care unit (ICU) admission, almost always as a consequence of respiratory failure [5]. Although no new cases have been reported since 8 September 2003 (http://www.who.int/csr/don/ 2003_09_24/en/; accessed 15 October 2003), the trans- 
missible nature of the disease and the high proportion of patients who require ICU admission means a recurrence of the epidemic may again result in a large number of critically ill patients with SARS. In order to be better prepared to both care for these patients and to carry out further research, it is useful to know the clinical course and outcome of the disease amongst those who require ICU and to know the factors which are associated with prognosis.

The overall mortality rate has not yet been confirmed but may be as high as $7-13 \%$ for younger patients and $40-55 \%$ for patients older than 60 years [6]. The overall case fatality ratio for probable SARS cases reported to the World Health Organization is $9.6 \%$ (http://www.who.int/ csr/sars/country/table2003_09_23/en/; accessed 15 October 2003). Data for critically ill patients is sparse and is limited to case series of 38 patients with SARS admitted to ICU in Toronto and 46 patients admitted to ICU in Singapore $[7,8]$.

Data from large series of patients, some of whom became critically ill, indicate that advanced age, high peak serum lactate dehydrogenase concentration, absolute neutrophilia, diabetes and other co-morbidities are independently associated with poor outcome $[5,9,10]$. There are few data specifically related to prognostic factors in critically ill patients with SARS. The Toronto study did not examine prognostic factors and the Singapore study only examined the relationship between respiratory parameters and outcome.

In view of the relative lack of data related to critically ill patients with SARS, we carried out a retrospective analysis of the first 54 patients admitted to our ICU with SARS to describe the clinical course and outcome of these patients and to carry out a preliminary investigation of factors associated with prognosis.

\section{Materials and methods}

We undertook, with institutional approval, a retrospective analysis of demographic, clinical, laboratory and radiological data on SARS cases admitted to our university hospital ICU from 12 March to 18 April 2003. During this period only patients suspected of having or confirmed to have SARS were admitted to the ICU.

\section{Definitions}

The definition of SARS used in our cohort was based on Centers for Disease Control and Prevention (CDC) criteria [11]: fever (temperature $>38^{\circ} \mathrm{C}$ ); radiological evidence of consolidation [by either plain radiograph or computerized tomographic (CT) scan of the thorax], with or without respiratory symptoms; and a history of exposure to a person with SARS, or direct contact with a person who later became ill with SARS.

The data collected and data definitions are given in Table 1. Data were collected until the day of discharge or death or for the first 28 days of the ICU stay, whichever was shorter. Physiological data were extracted from patient charts by a trained research nurse.
Microbiological data were prospectively collected by three ICU doctors and radiological data by two radiologists.

\section{Patient management}

Decisions regarding admission and management of individual patients were made by the individual intensive care and referring specialists, but there was broad consensus on a number of clinical issues.

Admission to the ICU was required when severe respiratory failure developed. Severe respiratory failure was evidenced by failure to maintain an arterial oxygen saturation of at least $90 \%$ while receiving supplemental oxygen of $50 \%$, and/or a respiratory rate greater than 35 breaths per minute. Criteria for intubation and positive pressure ventilation were persistent failure to achieve arterial oxygen saturation of $90 \%$ while receiving $15 \mathrm{l} / \mathrm{min}$ of oxygen via a non-rebreathing mask and/or onset of respiratory muscle fatigue as evidenced by an increase in $\mathrm{PaCO}_{2}$, sweating, tachycardia, tachypnoea and/or a subjective feeling of exhaustion.

A low-tidal-volume, low-pressure strategy was used for mechanical ventilation. The predominant modes of ventilation were volume control, pressure control, pressure-regulated volume control and pressure support. The low-tidal-volume protocol used in the Acute Respiratory Distress Syndrome Network study [12] was adapted to produce guidelines for setting tidal volume, driving pressure and respiratory rate (see electronic supplementary material for details). The target tidal volume was $4-6 \mathrm{ml} / \mathrm{kg}$ predicted body weight during controlled ventilation, but this was allowed to rise during the weaning phase. Positive end-expiratory pressure (PEEP) was set by titrating PEEP against oxygenation and compliance. In pressure-control mode the compliance was assessed from the measured tidal volume. In volume-control mode the compliance was assessed by measuring the plateau pressure. In pressureregulated volume control it was assessed from the driving pressure required to achieve the set tidal volume. Inspired oxygen concentration was titrated to achieve an arterial saturation of $88-94 \%$. Non-invasive positive pressure ventilation was avoided because of the potential risk of increased viral transmission resulting from mask leakage and high gas flow.

In general, there was a policy of using vasopressors to maintain an adequate blood pressure in preference to infusion of fluid and there was an attempt to keep patients in a fluid input/output balance of zero (excluding insensible loss).

Medical therapy evolved during the outbreak. The treatment of patients admitted early in the epidemic has been described previously [5] and was broadly similar to treatment outlined in a previous review [13], except that lower doses of ribavirin and higher doses of corticosteroids were used. Briefly, it consisted of broad-spectrum antibiotics, ribavirin and low-dose corticosteroids. Except in the initial phase of the epidemic, patients who deteriorated despite this treatment were given additional methylprednisolone in $500 \mathrm{mg}$ pulses up to a total of $3 \mathrm{~g}$. As the epidemic progressed, this maximum dose was increased to up to $5 \mathrm{~g}$ and in addition some patients were given convalescent serum donated by patients who had recovered from SARS and/or IgM-enriched immunoglobulin. Broad-spectrum antibiotics were administered at the time of institution of high-dose pulse methylprednisolone therapy. In the absence of clinical sepsis in the first 3-5 days following high-dose steroid therapy, antibiotics were withdrawn. Suspected infection was treated early with empirical broad spectrum antibiotics and, if necessary, anti-fungal agents. Antibiotics were modified according to bacterial culture of sputum, tracheal aspirate and blood. The dose of ribavirin was $8 \mathrm{mg} / \mathrm{kg}$ every $8 \mathrm{~h}$ intravenously for $7-10$ days followed by $4 \mathrm{mg} / \mathrm{kg}$ enterally for another 11-14 days. 
Table 1 Data collected from each patient and data definitions

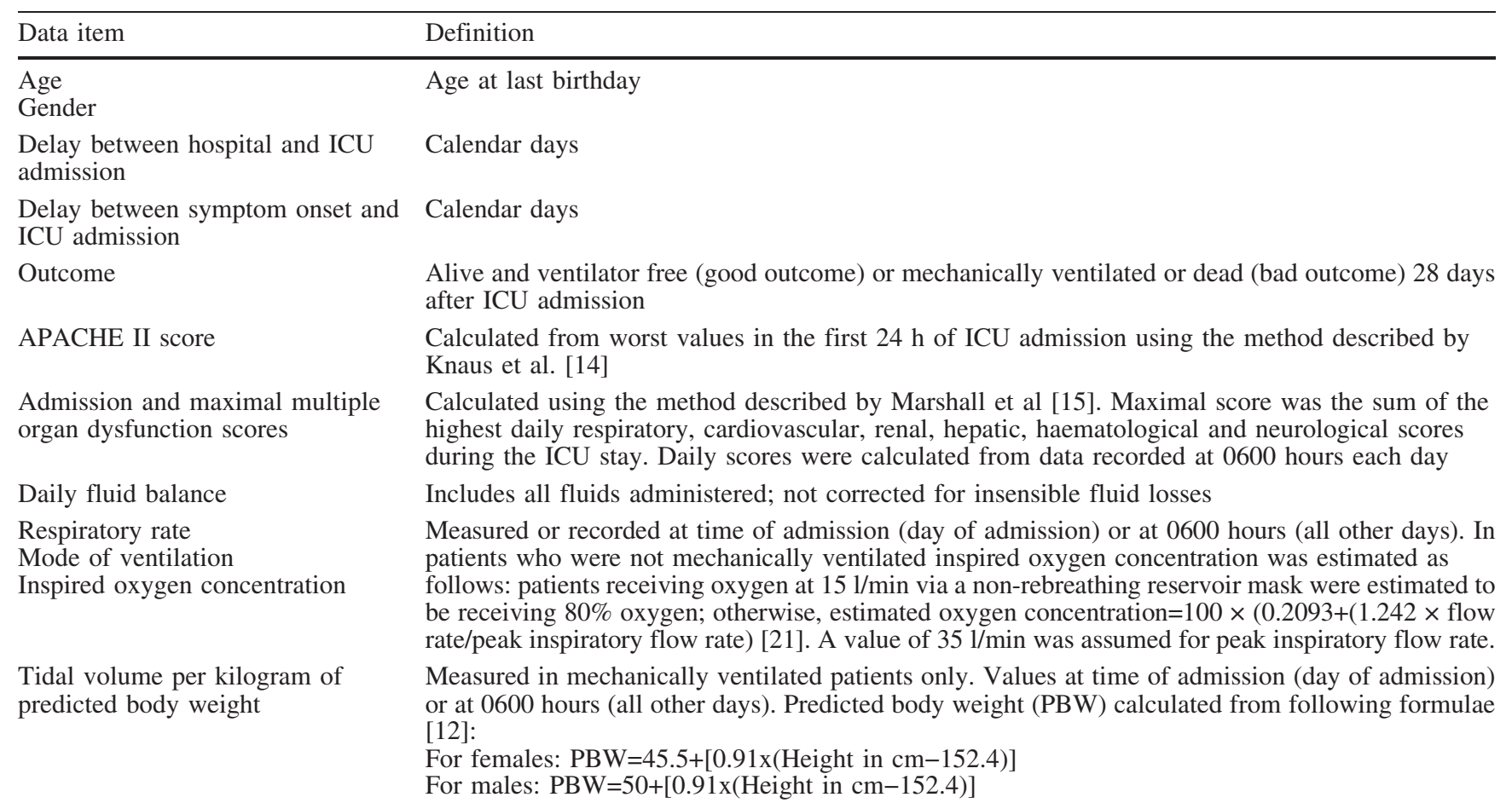

\section{Peak Paw}

Positive end-expiratory pressure

Presence of air leak from respiratory tract

Renal replacement therapy?

Inotropes or vasopressor?

Serum lactate dehydrogenase concentration

Lymphocyte count

Presence of acute respiratory distress syndrome

Total daily dose of corticosteroids

Nosocomial sepsis?

Bacteraemia?

Chronic disease or immuno suppression?

Independent in activities of daily living?
Pneumothorax, pneumomediastinum or surgical emphysema during ICU stay or first 28 days after ICU admission (whichever was shorter). Chest X-rays were performed daily and reviewed by a radiologist

Requirement for renal replacement therapy during ICU stay

Requirement for inotropes or vasopressors during ICU stay

Serum concentration at time of admission (day of admission) or at 0600 hours (all other days)

Blood lymphocyte count at time of admission (day of admission) or at 0600 hours (all other days)

Bilateral infiltrates on the chest X-ray, absence of left atrial hypertension and $\mathrm{PaO}_{2} / \mathrm{F}_{\mathrm{I}} \mathrm{O}_{2}$ ratio $<200 \mathrm{mmHg}$ [22]

Dose expressed as equivalents of methylprednisolone based on the following conversion:

$5 \mathrm{mg}$ hydrocortisone $=1 \mathrm{mg}$ methylprednisolone

$5 \mathrm{mg}$ prednisolone $=4 \mathrm{mg}$ methylprednisolone

Positive microbiological cultures and high enough clinical suspicion of infection to warrant treatment

For coagulase negative staphylococci: $\geq 2$ positive blood cultures; for all other organisms: $\geq 1$ positive blood culture

One or more of the following:

Heart failure. Systolic or diastolic dysfunction documented by history, examination and investigation (chest X-ray, echocardiogram, multi-gated acquisition scan or ventriculogram)

Chronic liver disease; clinical or biospy diagnosis of cirrhosis or other chronic liver disease such as chronic active hepatitis

Chronic renal impairment; history of chronic renal impairment or documented abnormal urea or creatinine

Cerebrovascular disease; stroke or transient ischaemic attack diagnosed clinically or documented by magnetic resonance imaging or computerized tomography

Active malignancy. Any malignancy except basal or squamous cell carcinoma of skin that was active on presentation or diagnosed $<1$ year before presentation

Immunosuppression; currently taking immunosuppressive drugs or suffering from an immunosuppressive disorder

Diabetes mellitus

Activities of daily living: eating; continence; voiding; transferring; bathing; dressing and walking 
Table 2 Ventilatory parameters. Values are expressed as mean (SD in parentheses). There were no significant differences between patients who did not develop an air leak on mechanical ventilation

\begin{tabular}{llll}
\hline & $\begin{array}{l}\text { Tidal volume }(\mathrm{ml} / \mathrm{kg} \text { predicted } \\
\text { body weight })\end{array}$ & Peak airway pressure $\left(\mathrm{cm} \mathrm{H}_{2} \mathrm{O}\right)$ & PEEP $\left(\mathrm{cm} \mathrm{H}_{2} \mathrm{O}\right)$ \\
\hline $\begin{array}{l}\text { Patients who did not develop an air leak } \\
\text { Patients who developed an air leak }\end{array}$ & $8.0(2.4)$ & $25.6(4.8)$ & $8.3(2.8)$ \\
Over the whole study period & $6.9(1.7)$ & $26.7(3.5)$ & $8.4(1.8)$ \\
Prior to development of leak & $6.8(1.7)$ & $26.5(3.1)$ & $9.6(2.9)$ \\
\hline
\end{tabular}

\section{Statistical analysis}

Continuous normally distributed data were compared using Student's unpaired $t$ test, categorical data using the $\chi^{2}$ test and ordinal or non-normally distributed continuous data using the Mann-Whitney U test. All statistical calculations were made using SPSS for Windows 11.5 (SPSS, Chicago, Ill.), Excel 2000 (Microsoft, Redmond, Wash.) or Hutchon's confidence interval calculator (http://www.hutchon.freeserve.co.uk/Wilsons.htm). A $p$ value $<0.05$ was considered significant. As the purpose of the univariate analysis of factors associated with outcomes was to generate hypotheses, no correction was made for multiple comparisons. After finding that fluid balance was associated with outcome, it was decided post hoc to further evaluate the relationship by analyzing the fluid balance in the first and second half of the patients' stay separately.

\section{Results}

The study population consisted of 54 patients with SARS who required admission to ICU. All patients were admitted for respiratory failure. Summary data for continuous normally distributed data are presented as mean and standard deviation, for categorical data as percentages and for ordinal data or non-normally distributed data as median and interquartile range. The mean age was 49.6 years (SD 16.9 years) and $57 \%$ of the patients were male. The median APACHE II score was 11 [interquartile range (IQR) 8-13], the mean $\mathrm{PaO}_{2} / \mathrm{F}_{\mathrm{I}} \mathrm{O}_{2}$ ratio on admission was 152 (SD 71) and the median admission multiple-organ dysfunction (MOD) score was 3 (IQR 2-4). The mean delay between hospital and ICU admission was 6.4 day (SD 6.0 days) and the mean delay between symptom onset and ICU admission was 9.6 days (SD 4.6 days).

At 28 days after ICU admission 34 patients $(63 \%$; $95 \%$ CI 49.6-74.6) were alive and not mechanically ventilated. Six patients were alive but still ventilated $(11.3 \%$; $95 \%$ CI 5.3-22.6) and 14 had died (25.9\%; CI 16.1-38.9). The median length of ICU stay was 8.5 days (IQR 5-22.3).

Forty-seven patients (87.0\%; 95\% CI 75.6-93.6) met criteria for the acute respiratory distress syndrome (ARDS) during their ICU stay and 27 (50.9\%; 95\% CI 37.9-63.9) required mechanical ventilation during their ICU admission. Of those who required mechanical ventilation 13 (48\%; CI 30.7-66.0) had died by 28 days. Mean tidal volume was $7.7 \mathrm{ml} / \mathrm{kg}$ predicted body weight (SD $2.2 \mathrm{ml} / \mathrm{kg}$ ) and mean PEEP was $8.3 \mathrm{~cm} \mathrm{H}_{2} \mathrm{O}$ (SD
$2.5 \mathrm{~cm} \mathrm{H}_{2} \mathrm{O}$ ). The median duration of mechanical ventilation was 14 days (IQR 8-25). Seven of 27 ventilated patients developed evidence of barotrauma (25.9\%; 95\% CI 13.2-44.7). Five patients developed a pneumothorax, accompanied in two cases by a pneumomediastinum. Two additional patients developed a pneumomediastinum without a pneumothorax. In most cases the pneumothorax or pneumomediastinum was accompanied by surgical emphysema. No patients developed surgical emphysema alone. There were no statistically significant differences in peak airway pressure, PEEP and tidal volume between those patients who developed an air leak on mechanical ventilation and those who did not (Table 2). One other patient developed a spontaneous pneumothorax while breathing unassisted and two others developed a pneumothorax following central venous cannulation.

Twenty-four patients (44.4\%; 95\% CI 32.0-57.6) required inotropes and $6(11.1 \%$; 95\% CI 5.2-22.2) required renal replacement therapy. Median maximal MOD score was 5 (IQR 3.3-9), of which the median respiratory component was 3 (IQR 3-4).

In the 39 patients who received pulsed methylprednisolone the mean $\mathrm{PaO}_{2} / \mathrm{F}_{\mathrm{I}} \mathrm{O}_{2}$ ratio (was 153 (SD 79) prior to the first pulse and 157 (SD 76) the following day. This difference was not statistically significant.

Results of univariate analysis of potential factors predicting poor outcome (death or mechanical ventilation 28 days after admission to ICU) are given in Table 3.

\section{Discussion}

The main findings of our study are as follows: firstly, in patients requiring ICU admission SARS is associated with considerable mortality and morbidity. Secondly, SARS predominantly causes respiratory failure. Thirdly, on univariate analysis advanced age, more severe illness, shorter delay between symptom onset and ICU admission, chronic disease or immunosuppression, lower steroid dose, higher lymphocyte count, nosocomial sepsis and positive fluid balance were associated with poor outcome.

At 28 days 20 of 54 patients (37\%) were either still mechanically ventilated or dead, indicating that SARS causes considerable mortality and morbidity, although the mortality is probably no greater than that associated with 
Table 3 Results of univariate analysis of potential prognostic factors. Data are presented as median (interquartile range) unless otherwise stated. NS not significant

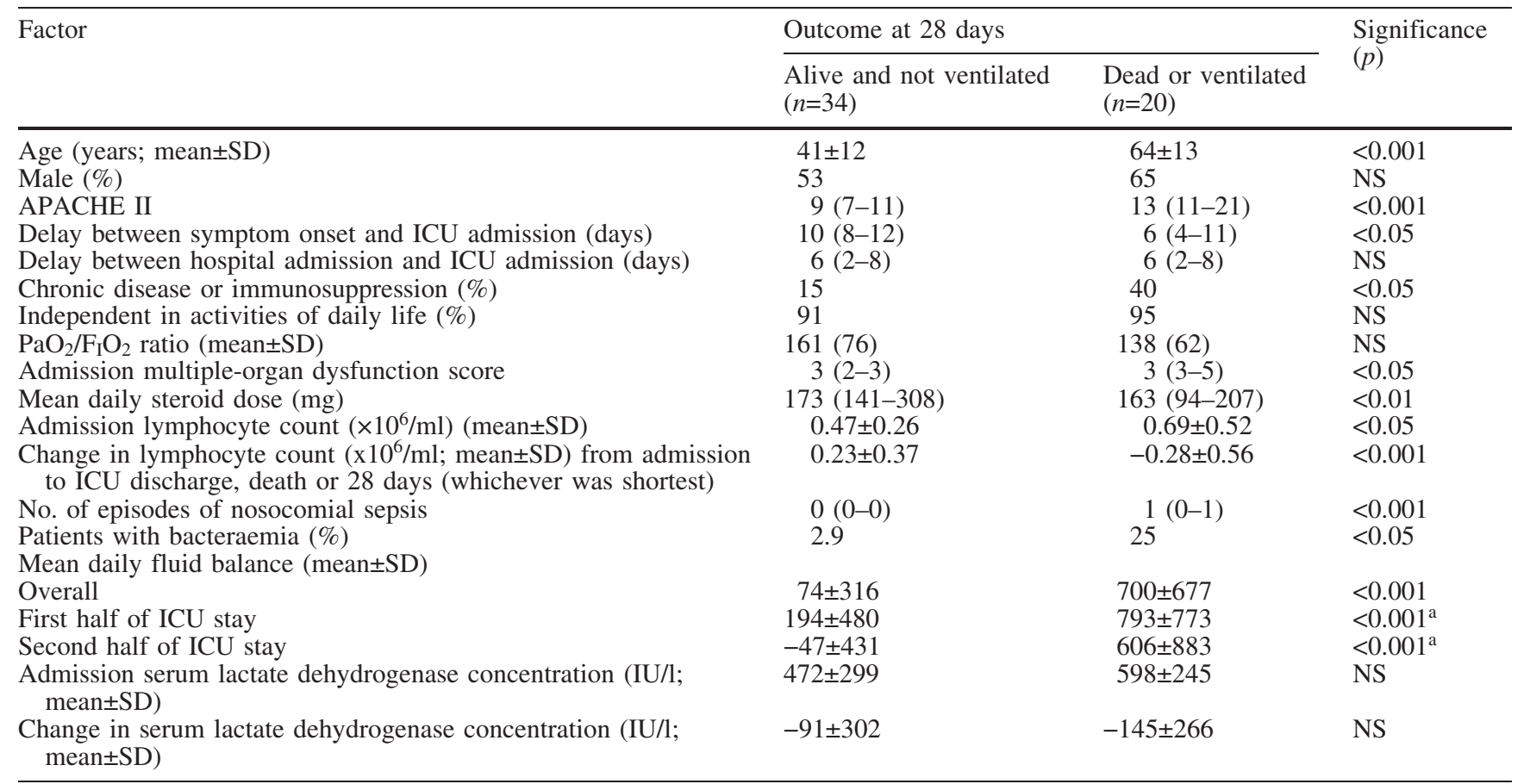

a Analysis of fluid balance by phase of ICU stay was carried out after initial univariate analysis showing that fluid balance was significantly associated with outcome

other forms of respiratory infection. Based on the APACHE II scores of our patients and the diagnostic category of respiratory failure due to infection, the predicted hospital mortality would be approximately $50 \%$ [14]. Similar results have been reported by other investigators with a 28-day ICU mortality of 34-37\% $[7,8]$. In the Toronto series an additional $16 \%$ of patients were alive but mechanically ventilated at 28 days [7].

The MOD score is a validated scoring system for quantifying multiple-organ dysfunction $[15,16]$. It is made up of six component scores (respiratory, cardiovascular, neurological, hepatic, renal and haematological) each of which is scored from 0-4, giving a maximum possible score of 24 . The median maximal MOD score in our patients was only 5 , of which the median respiratory component was 3 , indicating that SARS causes predominantly respiratory failure with little in the way of other organ failure. The large number of patients who required vasopressors or inotropes probably reflects our tendency to use these drugs instead of fluids to treat hypotension in patients with SARS. The severity of the respiratory failure is reflected by the fact that 47 of the 54 patients met criteria for ARDS at some stage in their ICU stay and the mean admission $\mathrm{PaO}_{2} / \mathrm{F}_{\mathrm{I}} \mathrm{O}_{2}$ ratio on admission to the ICU was only $152 \mathrm{mmHg}$. The incidence of barotrauma (25.9\%) was high compared with the $7-11 \%$ previously reported for ARDS $[17,12,18]$. Again, the Toronto and Singapore series show similar results with a 34 and $23 \%$ incidence of barotrauma despite the fact that in all three series the clinicians used low-volume, low-pressure strategies. Unfortunately, we did not record plateau pressures; however, the plateau pressure cannot exceed the peak airway pressure and the mean peak airway pressure prior to the development of an air leak was only $26.5 \mathrm{~cm} \mathrm{H} \mathrm{H}_{2} \mathrm{O}$. This suggests that the high incidence of air leaks was not due to excessive inflation pressures. Similarly, the mean tidal volume prior to development of an air leak was $6.79 \mathrm{ml} / \mathrm{kg}$ predicted body weight, which is similar to the values achieved in the low tidal volume group of the ARDSnet study [12]. This high incidence of adverse effects suggests that it may be preferable to avoid mechanical ventilation unless it is absolutely essential.

Univariate analysis demonstrated that a number of factors are associated with poor outcome in critically ill patients with SARS. It is likely that there was considerable confounding amongst these factors and that some of these factors would not be found to be independently associated with outcome on multivariate analysis. For example, both age and chronic disease are components of the APACHE II score [14] and both APACHE II and admission MOD score reflect severity of illness [15, 14]. In addition, the delay between symptom onset and admission to ICU is likely to reflect severity of illness with more severely ill patients deteriorating more rapidly and requiring earlier ICU admission. Unfortunately, the 
large number of potential prognostic factors and the relatively small size of our cohort prevented meaningful multivariate analysis [19]. Nevertheless, we believe the results of our analysis may be useful in generating hypotheses. At this stage of our knowledge of this new condition it is almost inevitable that observational studies will raise as many questions as they answer. They do, however, provide data that will help in the design of subsequent observational and interventional studies.

At first sight it may seem strange that the admission MOD score is reported as being significantly different in the good outcome group compared with the poor outcome group when the median score is 3 in both groups. Inspection of the interquartile ranges, however, reveals that the data for the good outcome group is skewed to the left while the data for the poor outcome group is skewed to the right.

The average daily dose of steroids was higher in patients who were alive and ventilator free at 28 days. High-dose pulse methylprednisolone was given to those patients who did not respond to smaller doses of steroids. Pulses were repeated if the initial response was considered inadequate. As a result, if steroids had no real effect, one would expect patients with a worse outcome to have received larger average daily doses of steroids as a physician response to their deteriorating condition. Instead, we found that patients with a good outcome received a higher average daily dose of steroids. However there are several confounding factors, such as duration of stay, and this finding should not be interpreted as proof of a beneficial effect of steroids particularly as there was no immediate effect of pulsed steroids on oxygenation.

The average daily recorded fluid balance was less positive in patients with a good outcome when measured over the entire study period and when the ICU stay (up to a maximum of 28 days) was divided into first and second halves. Given that the figures do not include an estimate of insensible fluid losses, these data suggest a marked negative actual fluid balance in patients with a good outcome and a slight positive fluid balance in patients with poor outcome. These data are in keeping with data suggesting that a restrictive fluid regime may be associated with better outcomes in ARDS [20]. It is, however, possible that the positive fluid balance in our patients with a poor outcome was due to the confounding effect of nosocomial sepsis. More patients in the poor-outcome group had nosocomial sepsis and patients with nosocomial sepsis are likely to receive more fluid due to the capillary leak and vasodilatation that characterizes systemic sepsis.

Surprisingly, patients with a good outcome had a lower lymphocyte count on admission to ICU than patients with a poor outcome. The lymphocyte count on admission to hospital has not been found to be associated with outcome and the explanation for our finding is unclear. More predictably, the lymphocyte count rose over the course of the ICU stay in those with a good outcome and fell in those with a poor outcome. If the change in lymphocyte count is found to be independently associated with outcome in these patients, this parameter may be a marker of disease activity. In contrast, the absence of a difference in the admission LDH concentration or in the change in LDH suggests that LDH is not a marker of disease activity, despite the fact that the LDH concentration on admission to hospital has been shown to be associated with outcome [5].

\section{Conclusion}

In conclusion, our data confirm previous data showing that SARS patients requiring ICU admission have considerable mortality. It mainly affects the lung, with little in the way of other organ failure, causing ARDS in most patients who require ICU admission. The incidence of barotrauma in those who require ventilation is high despite the use of low tidal volumes and peak airway pressures. Active diuresis and administration of higher doses of corticosteroids may be associated with better outcome but, in the absence of multivariate analysis, this suggestion should be treated with caution.

\section{References}

1. Centers for Disease Control and Prevention (2003) CDC update: outbreak of severe acute respiratory syndromeworldwide, 2003. Morbidity Mortality Weekly Rep 52:241-248

2. Peiris JS, Lai ST, Poon LL, Guan Y, Yam LY, Lim W, Nicholls J, Yee WK, Yan WW, Cheung MT, Cheng VC, Chan KH, Tsang DN, Yung RW, Ng TK, Yuen KY (2003) Coronavirus as a possible cause of severe acute respiratory syndrome. Lancet 361:1319-1325
3. Drosten C, Gunther S, Preiser W, van der WS, Brodt HR, Becker S, Rabenau H, Panning M, Kolesnikova L, Fouchier RA, Berger A, Burguiere AM, Cinatl J, Eickmann M, Escriou N, Grywna K, Kramme S, Manuguerra JC, Muller S, Rickerts V, Sturmer M, Vieth S, Klenk HD, Osterhaus AD, Schmitz H, Doerr HW (2003) Identification of a novel coronavirus in patients with severe acute respiratory syndrome. N Engl J Med 348:1967-1976
4. Fouchier RA, Kuiken T, Schutten M, Van Amerongen G, Van Doornum GJ, Van Den Hoogen BG, Peiris M, Lim W, Stohr K, Osterhaus AD (2003) Aetiology: Koch's postulates fulfilled for SARS virus. Nature 423:240

5. Lee N, Hui D, Wu A, Chan P, Cameron P, Joynt GM, Ahuja A, Yung MY, Leung CB, To KF, Lui SF, Szeto CC, Chung S, Sung JJY (2003) A major outbreak of severe acute respiratory syndrome in Hong Kong. N Engl J Med 348:1986-1994 
6. Donnelly CA, Ghani AC, Leung GM, Hedley AJ, Fraser C, Riley S, AbuRaddad LJ, Ho LM, Thach TQ, Chau P, Chan KP, Lam TH, Tse LY, Tsang T, Liu SH, Kong JH, Lau EM, Ferguson NM, Anderson RM (2003) Epidemiological determinants of spread of causal agent of severe acute respiratory syndrome in Hong Kong. Lancet 361:1761-1766

7. Fowler RA, Lapinsky SE, Hallett D, Detsky AS, Sibbald WJ, Slutsky AS, Stewart TE, for the Toronto SARS Critical Care Group (2003) Critically ill patients with severe acute respiratory syndrome. J Am Med Assoc 290:367373

8. Lew TWK, Kwek TK, Tai D, Ernest A, Loo S, Singh K, Kwan KM, Chan Y, Yim CF, Bek SL, Kor AC, Yap WS, Chelliah YR, Lai YC, Goh SK (2003) Acute respiratory distress syndrome in critically ill patients with severe acute respiratory syndrome. J Am Med Assoc 290:374-380

9. Booth CM, Matukas LM, Tomlinson GA, Rachlis AR, Rose DB, Dwosh HA, Walmsley SL, Mazzulli T, Avendano M, Derkach P, Ephtimios IE, Kitai I, Mederski BD, Shadowitz SB, Gold WL, Hawryluck LA, Rea E, Chenkin JS, Cescon DW, Poutanen SM, Detsky AS (2003) Clinical features and short-term outcomes of 144 patients with SARS in the greater Toronto area. J Am Med Assoc 289:2801-2809
10. Wong RSM, Wu A, To KF, Lee N, Lam CWK, Wong CK, Chan PKS, Ng MHL, Yu LM, Hui DS, Tam JS, Cheng G, Sung JJY (2003) Haematological manifestations in patients with severe acute respiratory syndrome: retrospective analysis. Br Med J 326:1358

11. Centers for Disease Control and Prevention (2003) Updated interim US case definition for severe acute respiratory syndrome (SARS)-update, 30 April 2003 http://www.cdc.gov/ncidod/ sars/casedefinition.htm

12. The Acute Respiratory Distress Syndrome Network (2000) Ventilation with lower tidal volumes as compared with traditional tidal volumes for acute lung injury and the acute respiratory distress syndrome. N Engl J Med 342:13011308

13. Lapinsky SE, Hawryluck L (2003) ICU management of severe acute respiratory syndrome. Intensive Care Med 29:870875

14. Knaus WA, Draper EA, Wagner DP, Zimmerman JE (1985) APACHE II: a severity of disease classification. Crit Care Med 13:818-829

15. Marshall JC, Cook DJ, Christou NV, Bernard GR, Sprung CL, Sibbald WJ (1995) Multiple organ dysfunction score: a reliable descriptor of a complex clinical outcome. Crit Care Med 23:1638-1652

16. Buckley TA, Gomersall CD, Ramsay SJ (2003) Validation of the multiple organ dysfunction (MOD) score in critically ill medical and surgical patients. Intensive Care Med 29:2216-2222
17. Weg JG, Anzueto A, Balk RA, Wiedemann HP, Pattishall EN, Schork MA, Wagner LA (1998) The relations of pneumothorax and other air leaks to mortality in the acute respiratory distress syndrome. N Engl J Med 338:341346

18. Stewart TE, Meade MO, Cook DJ, Granton JT, Hodder RV, Lapinsky SE, Mazer CD, McLean RF, Rogovein TS, Schouten D, Todd TRJ, Slutsky AS, the Pressure- and Volume-Limited Ventilation Strategy Group (1998) Evaluation of a ventilation strategy to prevent barotrauma in patients at high risk for acute respiratory distress syndrome. $\mathrm{N}$ Engl J Med 338:355-361

19. van Walraven C, Hébert PC (1996) A reader's guide to the evaluation of prognostic studies. Postgrad Med J 72:6-11

20. Schuller D, Mitchell JP, Calandrino FS, Schuster DP (1991) Fluid balance during pulmonary edema. Is fluid gain a marker or a cause of poor outcome? Chest 100:1068-1075

21. Leigh JM (1990) Oxygen therapy. In: Scurr C, Feldman S, Soni N (eds) Scientific foundations of anaesthesia, 4th edn. Heinemann Medical Books, Oxford, pp 287-296

22. Bernard GR, Artigas A, Brigham KL, Carlet J, Falke KJ, Hudson LD, Lamy M, LeGall JR, Morris A, Spragg R, the Consensus Committee (1994) The American-European consensus conference on ARDS. Am J Respir Crit Care Med 149:818-824 